\title{
Identification and characterization of dermatophyte species and strains with PCR amplification
}

\author{
GUOFANG LIU $^{1,2}$, CHENGHUA HE ${ }^{2}$ and HAIBIN ZHANG ${ }^{1}$ \\ ${ }^{1}$ College of Veterinary Medicine, Nanjing Agricultural University, Nanjing, Jiangsu 210095; \\ ${ }^{2}$ Department of Animal Husbandry and Veterinary Medicine, Jiangsu Polytechnic College of \\ Agriculture and Forestry, Zhenjiang, Jiangsu 212400, P.R. China
}

Received November 18, 2013; Accepted May 13, 2014

DOI: $10.3892 /$ etm.2014.1785

\begin{abstract}
The aim of the present study was to use two polymerase chain reaction (PCR) methods, with (GACA) $)_{4}$ and non-transcribed spacer (NTS) as primers, to identify and characterize dermatophyte isolates from dogs and cats to a species and strain level. A total of 45 isolates from nine dermatophyte species were collected from pet dogs and cats and subjected to PCR amplification with the microsatellite primer (GACA) 4 . Dermatophyte strains of three of the same species collected from four cities were subjected to PCR amplification with the NTS primer set. These two PCR methods were applied to identify and characterize the dermatophyte isolates to a species and strain level. Regional differences among the strain specificities were also examined. The results from PCR with $(\mathrm{GACA})_{4}$ demonstrated that strains from the same species produced similar PCR product band patterns. In addition, these patterns differed among species, indicating that $(\mathrm{GACA})_{4}$ primer-based PCR was able to distinguish between the various dermatophyte species. By contrast, dermatophyte isolates and/or strains within the same species revealed various band patterns with NTS-based PCR. In addition, the results indicated that regional differences contributed to the variations in PCR product band patterns. Therefore, the results of the present study indicate that the NTS-based PCR method is efficient in distinguishing dermatophytes to the strain level, while a combination of (GACA $)_{4}$ and NTS primer-based PCR methods is able to clarify dermatophyte isolates to a species and strain level. The present study provides information concerning the identification of pathogenic fungi and the epidemiological characteristics of fungal skin diseases.
\end{abstract}

Correspondence to: Dr Haibin Zhang, College of Veterinary Medicine, Nanjing Agricultural University, 1 Weigang, Nanjing, Jiangsu 210095, P.R. China

E-mail: mf_liuguofang@hotmail.com

Key words: dermatophyte, polymerase chain reaction, microsatellite primer, non-transcribed spacer

\section{Introduction}

Fungal infections affect superficial keratinized tissues, including the skin, hair and nails, in humans and animals resulting in difficult-to-treat dermatosis. Fungi derived from pet dogs and cats, including dermatophytes (Deuteromycotina, Hyphomycetes, Hyphomycetales, Moniliaceae, Trichophyton, Microsporum and Epidermophyton), Malassezia, Saccharomycetes (mainly Candida) and non-dermatophyte molds (Scopulariopsis, Aspergillus and Fusarium), are also able to infect human skin (1). The routine identification and classification of these skin-infecting fungi is mainly based on clinical symptoms and the morphological and/or biochemical characteristics of the fungi. In recent years, molecular approaches, including pulsed-field gel electrophoresis, random amplified polymorphic DNA analysis, polymerase chain reaction (PCR) amplification using NTS and internal transcribed spacer (ITS) primers (2), nested-PCR, PCR-restriction fragment length polymorphism (RFLP) analysis, arbitrary primer PCR and ITS region sequencing (3), have been used for the identification of dermatophyte species and strains.

The simple repetitive oligonucleotide, $(\mathrm{GACA})_{4}$, is a highly variable microsatellite that has been used as a PCR primer for the efficient identification of skin tinea infections and pathogenic Candida species (4). PCR using (GACA) has also been used for the classification and identification of human pathogenic fungi (5). In the present study, microsatellite $(\mathrm{GACA})_{4}$ and non-transcribed spacer (NTS) primers were used to perform PCR amplification with the aim of identifying and characterizing dermatophyte isolates from dogs and cats to a species and strain level.

\section{Materials and methods}

Fungal strains. Pathogenic fungal strains were isolated from pet dogs and cats. In total, 45 strains were analyzed for species identification and characterization, including five strains from each of the following species: Trichophyton rubrum (T. rubrum), Trichophyton mentagrophytes (T. mentagrophytes), Epidermophyton floccosum (E. floccosum), Microsporum canis (M. canis), Microsporum gypseum (M. gypseum), Candida albicans (C. albicans), Candida 
Table I. PCR primer sets.

\begin{tabular}{lcc}
\hline Regions & Primers & \multicolumn{1}{c}{ Sequences } \\
\hline NTS-1 & TrNTSF-2 & 5'-ACC GTA TTA AGC TAG CGC TGC-3' \\
& TrNTSR-4 & 5'-TGC CAC TTC GAT TAG GAG GC-3' \\
NTS-2 & TrNTSR-1 & 5'-CTC AGT CGA ACC GTG AGG C-3' \\
& TrNTSC-1 & 5'-CGA GAC CAC GTG ATACAT GCG-3'
\end{tabular}

PCR, polymerase chain reaction; NTS, non-transcribed spacer.

tropicalis (C. tropicalis), Candida glabrata (C. glabrata) and Candida parapsilosis (C.parapsilosis).

For strain comparison, 54 strains of T. rubrum, 26 strains of T. mentagrophytes and 32 strains of $M$. canis were collected from four cities, namely Nanjing, Wuxi, Shanghai and Hangzhou in China.

The strains were cultured in Sabouraud dextrose agar medium at $27^{\circ} \mathrm{C}$ with a humidity of $95 \%$ in a mold incubator. Filamentous fungi were usually cultured for 2 weeks, whereas yeasts were cultured for 2-3 days.

DNA extraction and purification. Fungal genomic DNA was extracted using the benzyl chloride extraction method and purified with phenol-chloroform as previously described $(6,7)$. Growing fungi were harvested through filtration and washed three times with sterile saline. The samples were transferred to $1.5-\mathrm{ml}$ microcentrifuge tubes and subjected to centrifugation at $5,700 \mathrm{x} \mathrm{g}$ at room temperature for $1 \mathrm{~min}$. Next, $500 \mu \mathrm{l}$ extraction buffer $[100 \mathrm{mM}$ Tris- $\mathrm{HCl}(\mathrm{pH} 9.0)$ and $40 \mathrm{mM}$ EDTA], $100 \mu l$ sodium dodecyl sulfate (10\%) and $300 \mu \mathrm{l}$ benzyl chloride (Sinochem Ningbo Chemicals Co., Ltd., Ningbo, China) were added. The mixture was vortexed and incubated at $50^{\circ} \mathrm{C}$ for $3 \mathrm{~min}$ with mild shaking. Following centrifugation at $6,000 \mathrm{xg}$ at a temperature of $4^{\circ} \mathrm{C}$ for $10 \mathrm{~min}$, the supernatant was collected in a new tube. Next, $300 \mu \mathrm{l}$ sodium acetate $(3 \mathrm{M})$ was added and the samples were mixed and centrifuged again at $6,000 \mathrm{x} \mathrm{g}$ at $4^{\circ} \mathrm{C}$ for $10 \mathrm{~min}$. Following aspiration, the supernatant was transferred into a tube containing $500 \mu \mathrm{l}$ isopropanol. The sample was stored at $-70^{\circ} \mathrm{C}$ for $1 \mathrm{~h}$ and DNA was precipitated following centrifugation at $6,000 \mathrm{x}$ g at $4^{\circ} \mathrm{C}$ for $10 \mathrm{~min}$. Extracted DNA was treated with RNase A and then with phenol/chloroform/isoamyl alcohol (v:v:v, 25:24:1; all purchased from Sinochem Ningbo Chemicals Co., Ltd.). Following 2 or 3 centrifugations, DNA was precipitated with ice-cold pure ethanol, washed with $70 \%$ ethanol, air-dried and then resuspended in Tris-EDTA buffer for additional study. Yeast genomic DNA was prepared using a quick DNA extraction kit (Shanghai Huashun Bioengineering Co., Ltd., Shanghai, China), according to the manufacturer's instructions.

PCR amplification. PCR amplification was conducted in volumes of $100 \mu \mathrm{l}$ containing $10 \mathrm{mM}$ Tris- $\mathrm{HCl}(\mathrm{pH}$ 9.0), $0.1 \%$ Triton X-100, $50 \mathrm{mM} \mathrm{KCl,} 1.5 \mathrm{mM} \mathrm{MgCl} 2,200 \mu \mathrm{M}$ dATP, dCTP, dGTP and dTTP, 5 units Taq DNA polymerase (Takara Bio, Inc., Dalian, China), $2.5 \mu \mathrm{M}$ primer and $20 \mathrm{ng}$ template DNA. Primer sequences are shown in Table I (synthesized by Shanghai Yingweijie Co., Shanghai, China). PCR amplification was performed in a GeneAmp PCR System 9600 (Perkin-Elmer, Norwalk, CT, USA). Samples were first heated at $94^{\circ} \mathrm{C}$ for $4 \mathrm{~min}$, followed by 35 cycles of $92^{\circ} \mathrm{C}$ for $1 \mathrm{~min}$, $55^{\circ} \mathrm{C}$ for $1 \mathrm{~min}$ (NTS-1 primers) or $58^{\circ} \mathrm{C}$ for $1 \mathrm{~min}$ (NTS-2 primers) and $72^{\circ} \mathrm{C}$ for $2 \mathrm{~min}$, prior to an extension step at $72^{\circ} \mathrm{C}$ for $10 \mathrm{~min}$. For amplification using $(\mathrm{GACA})_{4}, \mathrm{PCR}$ was carried out for 39 cycles of denaturation at $93^{\circ} \mathrm{C}$ for $1 \mathrm{~min}$, annealing at $50^{\circ} \mathrm{C}$ for $1 \mathrm{~min}$, and extension at $72^{\circ} \mathrm{C}$ for $1 \mathrm{~min}$, followed by a final extension step at $72^{\circ} \mathrm{C}$ for $7 \mathrm{~min}$. The products were electrophoresed in $2 \%$ agarose gels and detected using a gel imaging analysis system (Bio-Rad, Hercules, CA, USA).

Statistical analysis. Data were analyzed using SPSS software, version 10.0 (SPSS, Inc., Chicago, IL, USA). Differences were compared with the $\chi^{2}$ test and $\mathrm{P}<0.05$ was considered to indicate a statistically significant difference.

\section{Results}

Identification of dermatophyte species using $(G A C A)_{4}$ primer-based PCR. PCR, with the short oligonucleotide $(\mathrm{GACA})_{4}$ as a primer, was performed to identify and characterize dermatophytes. DNA products were determined by gel electrophoresis and image analysis. The results showed that the PCR product bands ranged between 300 and 3,000 bp (Fig. 1). Based on clinical phenotypic analysis, strains from the same species produced similar patterns, but these patterns changed from species to species (Fig. 1). These results indicate that $(\mathrm{GACA})_{4}$ primer-based PCR is able to distinguish between various dermatophyte species, which may be useful for species identification.

Characterization of dermatophyte strains using NTS-based $P C R$. To determine intraspecies variation and identify dermatophyte isolates to the strain level, PCR amplification was performed with NTS-1 and NTS-2 primer sets. The dermatophyte isolates were collected from four cities in China. As shown in Fig. 2, the NTS-1 amplification products from 54 T. rubrum strains were divided into five patterns (A, B, C, $\mathrm{D}$ and $\mathrm{E}$ ) with typical fragment sizes of 250, 550, 750, 900 and 1,000 bp, respectively (Fig. 2). By contrast, the NTS-2 amplification products exhibited two band patterns (I and II) with typical fragment sizes of 500 and $450 \mathrm{bp}$, respectively (Fig. 3).

The NTS-1-based PCR amplification products from the strains of T. mentagrophytes were divided into four patterns (A, B, C and D) with typical fragment sizes of 850,900 , 
A

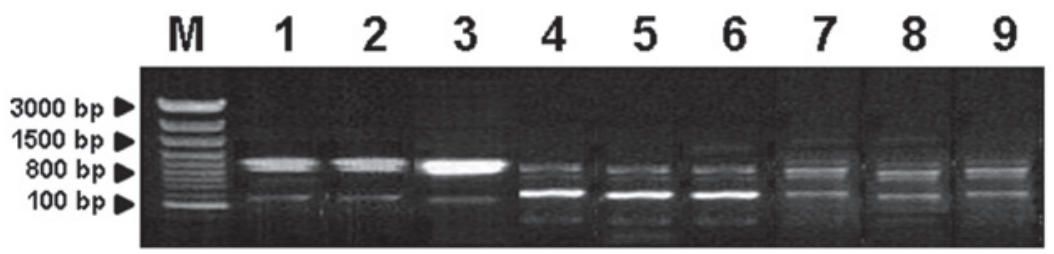

B

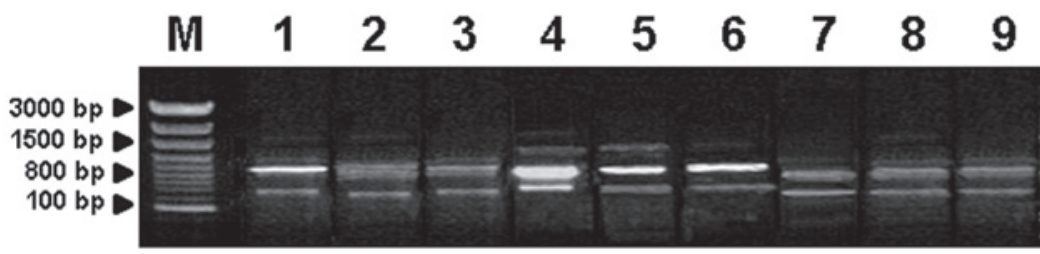

C

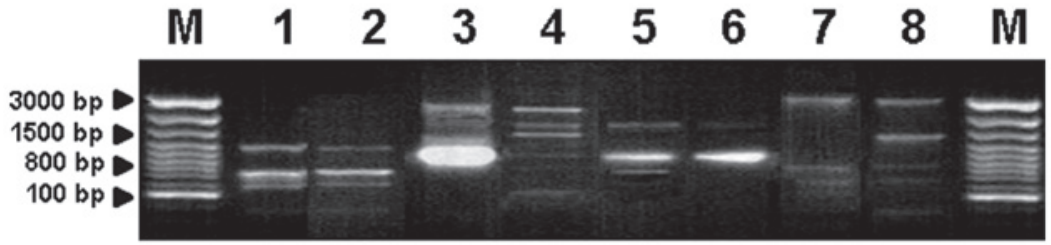

D

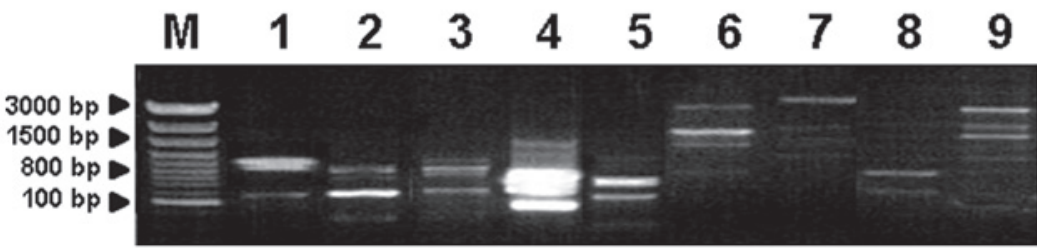

Figure 1. Identification of dermatophyte species using (GACA) $)_{4}$ primer-based PCR. PCR products of various dermatophyte isolates are shown. Lanes: M, molecular weight marker; (A) 1-3, T. rubrum; 4-6, T. mentagrophytes; 7-9, M. canis; (B) 1-3, M. canis; 4-6, M. gypseum; 7-9, E. floccosum; (C) 1 and 2 , C. albicans; 3 and 4, C. glabrata; 5 and 6,C.tropicalis; 7 and 8,C.parapsilosis; (D) 1, T. rubrum; 2, T. mentagrophytes; 3, M. canis; 4, M. gypseum; 5, E. floccosum; 6 , C.albicans; 7, C. parapsilosis; 8, C. tropicalis; 9, C. glabrata. PCR, polymerase chain reaction; T. rubrum, Trichophyton rubrum; T. mentagrophytes, Trichophyton mentagrophytes; M. canis, Microsporum canis; M. gypseum, Microsporum gypseum; E. floccosum, Epidermophyton floccosum; C. albicans, Candida albicans; C. glabrata, Candida glabrata; C. tropicalis; Candida tropicalis; C. parapsilosis; Candida parapsilosis.

$\begin{array}{lllllllllllllllllllllllllllll}M & 1 & 2 & 3 & 4 & 5 & 6 & 7 & 8 & 9 & 10 & 11 & 12 & 13 & 14 & 15 & 16 & 17 & 18 & 19 & 20 & 21 & 22 & 23 & 24 & 25 & 26 & 27 & M\end{array}$

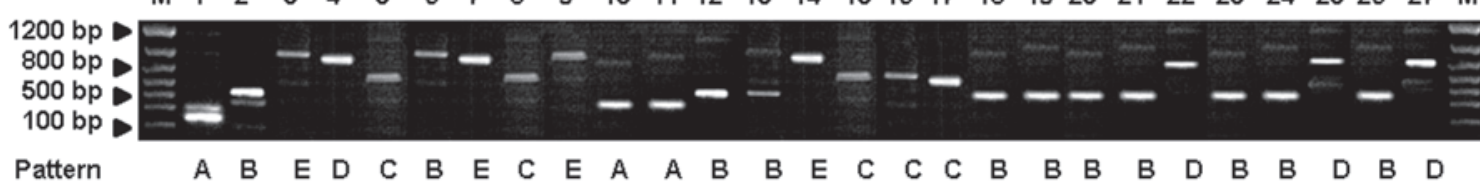

$\begin{array}{lllllllllllllllllllllllllllll}\text { M } & 28 & 29 & 30 & 31 & 32 & 33 & 34 & 35 & 36 & 37 & 38 & 39 & 40 & 41 & 42 & 43 & 44 & 45 & 46 & 47 & 48 & 49 & 50 & 51 & 52 & 53 & 54 & M\end{array}$

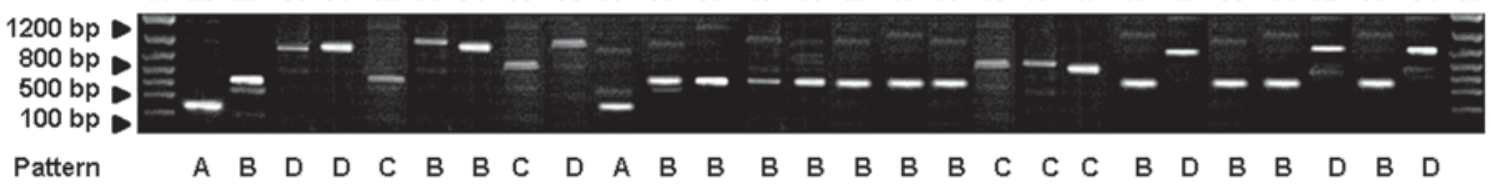

Figure 2. Characterization of 54 T. rubrum strains using PCR with the NTS-1 primer sets. Patterns were designated arbitrarily as A, B, C, D and E. Lanes: M, molecular weight marker; 1-54, 54 T. rubrum strains collected from four cities. PCR, polymerase chain reaction; NTS, non-transcribed spacer; T. rubrum, Trichophyton rubrum.

1,100 and 1,200 bp, respectively (Fig. 4A). In addition, the NTS-2-based PCR amplification products were divided into two patterns (I and II) with typical fragment sizes of 800 and 650 bp, respectively (Fig. 4B). For M. canis, the NTS-1 amplification products were divided into four patterns (A, B, $\mathrm{C}$ and D) with typical fragment sizes of 1,100, 1,000, 800 and 750 bp, respectively (Fig. 5A). However, the NTS-2 amplification products for the various strains exhibited the same profile, consisting of two clearly distinguishable bands of 800 and $650 \mathrm{bp}$ (Fig. 5B). These results indicate that dermatophyte isolates and/or strains within the same species exhibit various band patterns with NTS-based PCR, indicating that this method may be a useful tool to identify dermatophytes to the strain level.

Regional differences in the NTS-based PCR product band patterns from the same dermatophyte species. To investigate the regional differences among dermatophyte strains, NTS-based 
Table II. Classification of NTS-1-based PCR amplification band patterns for T. rubrum.

\begin{tabular}{lccccc}
\hline Location & Pattern A, n (\%) & Pattern B, n (\%) & Pattern C, n (\%) & Pattern D, n (\%) & Pattern E, n (\%) \\
\hline Nanjing & $1(5.26)$ & $12(63.16)$ & $2(10.53)$ & $2(10.53)$ & $2(10.53)$ \\
Wuxi & $3(20.00)$ & $8(53.33)$ & $1(6.67)$ & $1(6.67)$ & $2(13.33)$ \\
Hangzhou & $1(8.33)$ & $3(25.00)$ & $2(16.67)$ & $6(50.00)$ & $0(0.00)$ \\
Shanghai & $0(0.00)$ & $1(12.5)$ & $5(62.50)$ & $1(12.50)$ & $1(12.5)$ \\
Total & $5(9.26)$ & $24(44.44)$ & $10(18.52)$ & $10(18.52)$ & $5(9.26)$ \\
\hline
\end{tabular}

NTS, non-transcribed spacer; PCR, polymerase chain reaction; T. rubrum, Trichophyton rubrum.

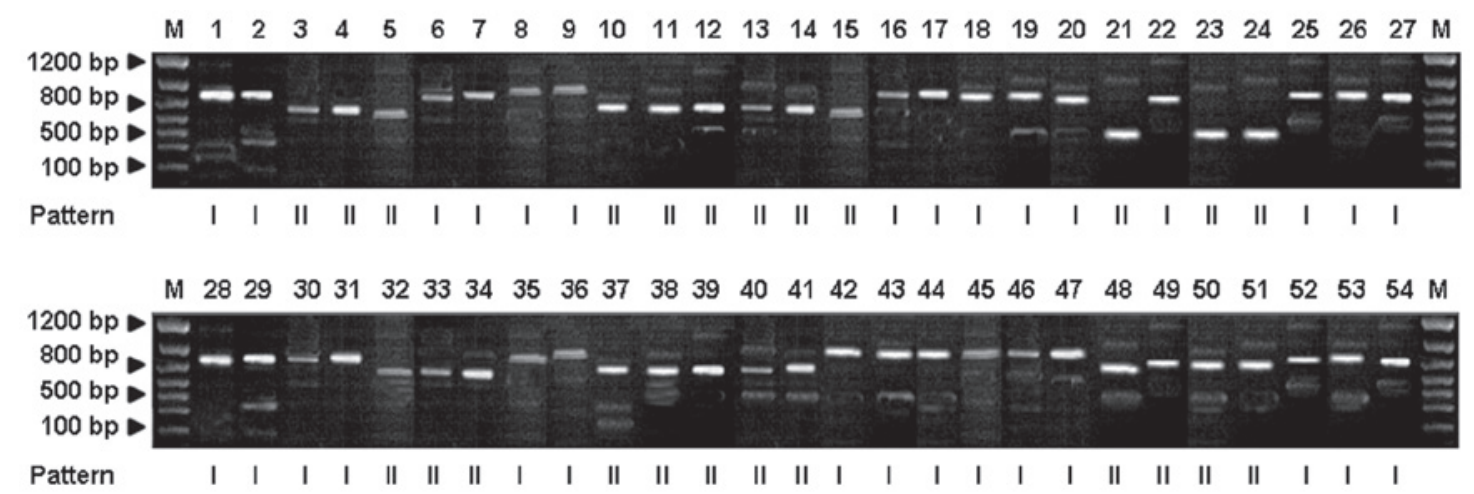

Figure 3. Characterization of 54 T.rubrum strains using PCR with NTS-2 primer sets. Patterns were designated arbitrarily as I and II. Lanes: M, molecular weight marker; 1-54, 54 T. rubrum strains collected from four cities. PCR, polymerase chain reaction; NTS, non-transcribed spacer; T. rubrum, Trichophyton rubrum.

A $\begin{array}{llllllllllllllllllllllllllll}M & 1 & 2 & 3 & 4 & 5 & 6 & 7 & 8 & 9 & 10 & 11 & 12 & 13 & 14 & 15 & 16 & 17 & 18 & 19 & 20 & 21 & 22 & 23 & 24 & 25 & 26 & M\end{array}$

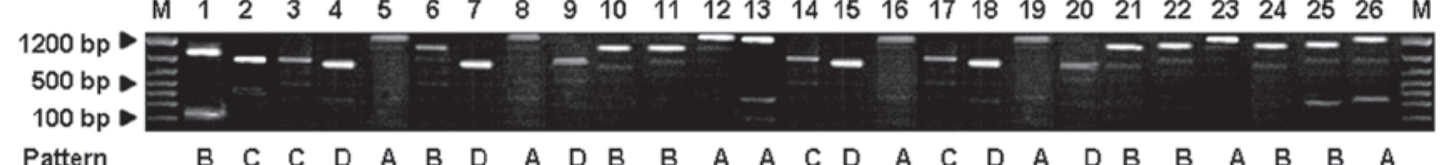

B

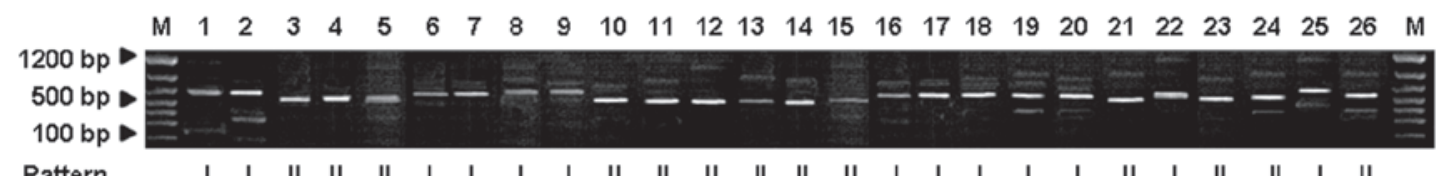

Figure 4. Characterization of 26 T. mentagrophytes strains using NTS-based PCR. PCR products with (A) NTS-1 primer sets (patterns were designated arbitrarily as A, B, C and D) and (B) NTS-2 primer sets (patterns were designated arbitrarily as I and II). Lanes: M, molecular weight marker; 1-26, 26 T. mentagrophytes strains collected from four cities. PCR, polymerase chain reaction; NTS, non-transcribed spacer; T. mentagrophytes, Trichophyton mentagrophytes.

A

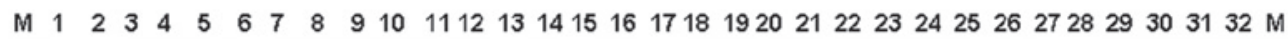

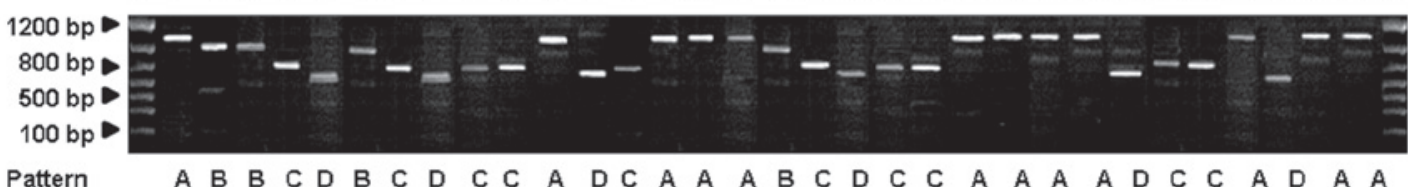

B

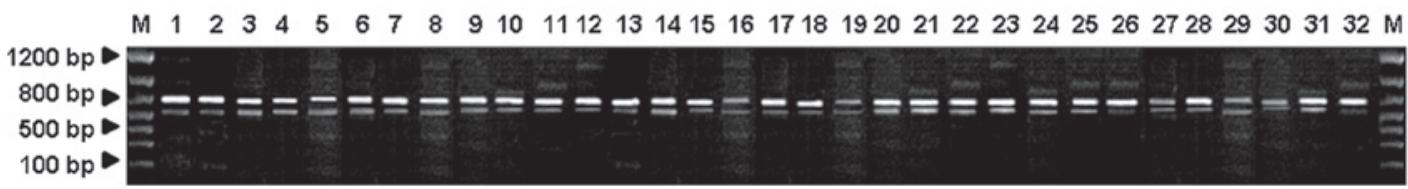

Figure 5. Characterization of 32 M. canis strains using NTS-based PCR. PCR products with (A) NTS-1 primer sets (patterns were designated arbitrarily as A, B, C and D) and (B) NTS-2 primer sets. Lanes: M, molecular weight marker; 1-32, $32 \mathrm{M}$. canis strains collected from four cities. PCR, polymerase chain reaction; NTS, non-transcribed spacer; M. canis, Microsporum canis. 
PCR band pattern percentages were analyzed. Intraspecies classification of the NTS-1-based PCR amplification band patterns of 54 T. rubrum strains is shown in Table II. In Nanjing and Wuxi, pattern B accounted for a relatively large proportion when compared with the other four band patterns. By contrast, patterns D and C were predominant in Hangzhou and Shanghai, respectively. For NTS-2-based PCR amplification, 30 of the 54 T. rubrum strains were classified as pattern I, accounting for $55.56 \%$. The remaining 24 strains were classified as pattern II, accounting for $44.44 \%$ (Fig. 3). The majority of strains from Nanjing and Wuxi were classified as pattern I, while strains from Hangzhou and Shanghai were primarily classified as pattern II.

For M. canis, patterns A and C of the NTS-1-based PCR amplification product bands accounted for 37.50 and $31.25 \%$ of strains, respectively. There were no regional differences for M. canis in patterns A and C of the NTS-1-based PCR amplification product bands. The NTS-1 and NTS-2-based PCR amplification band patterns of T. mentagrophytes exhibited no statistically significant differences among the various regions, although the incidence of pattern I strains in Wuxi and Nanjing was slightly higher than that in the other locations. Therefore, these results indicate that regional differences contribute to variations in PCR product band patterns, indicating that NTS-based PCR may be efficient in distinguishing dermatophytes to the strain level.

\section{Discussion}

At present, skin fungal classification and identification is primarily based on the clinical symptoms and characteristics of in vitro culture. However, this is a time-consuming process that is not able to identify dermatophyte strains. In addition, accuracy and precision is easily affected by culture conditions and environmental factors. In the present study, $(\text { GACA })_{4}$ and NTS primer-based PCR methods were applied to identify dermatophyte isolates to a species and strain level. The results revealed significant differences in the (GACA) primer-based PCR amplification band patterns among the tested 45 clinical dermatophyte isolates. Band patterns were clear with specific distributions, rendering them distinguishable. The PCR product band patterns of the nine species were similar to those described by Zhu et al (5), with an extra $1,500 \mathrm{bp}$ fragment produced in amplification. However, further experiments are required to confirm whether this difference is attributed to the various origins of these dermatophyte strains.

The characteristics of ITS- and NTS-based PCR amplification patterns have been applied to study fungal species specificities $(4,8)$. An ITS is a relatively conserved gene sequence involved in species specificity of dermatophytes (9). The 18, 5.8 and $25 \mathrm{~S}$ gene fragments of ITS1 and ITS2 from dermatophytes can be PCR-amplified using NTS 9 and ITS 6 as primers (10). The ITS regions of 37 T. mentagrophytes strains have been sequenced and divided into three homology groups and intraspecies specificity has also been analyzed. Jackson et al classified T. rubrum into 14 types using probes designed by the sequences of $18 \mathrm{~S}$ rDNA and ITS regions (11). The majority of strains fell into four types with evident polymorphisms. However, the methods used by Jackson et al were rather complex.
An NTS is a highly variable region; thus, its sequence is ideal for distinguishing between species and/or strains. Mochizuki et al divided T. rubrum into five types, according to the RFLP analysis of NTS (12). Jackson et al also confirmed the specificity, reproducibility and stability of NTS (11). A common method to distinguish between the species of T. mentagrophytes and M. canis uses ITS regions and random primers (13-16). Jackson et al amplified the ITS regions of 17 dermatophyte strains and digested the samples with MvaI. A total of 13 fragments were produced. One band was specifically produced in each of the nine fungi, including $M$. canis, M. gypseum and T. mentagrophytes, indicating that the MvaI digestion reaction only partly reflects the inter- and intraspecific specificities. Although NTS-2 has no specificity within the $M$. canis species, the specificities of ITS and NTS may be used to classify T. mentagrophytes, M. canis and T. rubrum derived from dogs and cats. ITS and NTS have been further demonstrated to be specific in the classification of pathogenic fungi (17). Despite the efficiency of DNA sequencing in fungal species classification, the complexity and high cost limits its application. RFLP analysis of ITS and NTS in rDNA is simple and the results are stable (18). This procedure is easily and widely applicable to the identification of fungal species and is important for the study of fungal epidemiology.

The results of the present study indicate that regional differences contribute to variations in PCR product bands of dermatophyte strains, and possess the potential to distinguish dermatophytes to the strain level. However, for T. rubrum, the pattern distribution in the current study was not consistent with the six-type classification by Fan et al (19) and the 14-type classification by Jackson et al (21). This discrepancy may be associated with sample insufficiency and the limited sampling regions. Furthermore, the NTS-based PCR amplification product band patterns of T. mentagrophytes and $M$. canis did not exhibit differences among regions as clearly as those in T. rubrum. Therefore, more samples are required to verify whether significant regional differences exist in the NTS-based PCR amplification product band patterns of these dermatophyte species.

In conclusion, using (GACA) $)_{4}$ and NTS as primers, PCR was accurately, conveniently and efficiently performed to clarify dermatophyte isolates to a species and strain level. The present study provides information concerning the identification of pathogenic fungi and the epidemiological characteristics of fungal skin diseases.

\section{References}

1. Moretti A, Agnetti F, Mancianti F, et al: Dermatophytosis in animals: epidemiological, clinical and zoonotic aspects. $\mathrm{G}$ Ital Dermatol Venereol 148: 563-572, 2013.

2. Gaedigk A, Gaedigk R and Abdel-Rahman SM: Genetic heterogeneity in the rRNA gene locus of Trichophyton tonsurans. J Clin Microbiol 41: 5478-5487, 2003.

3. Kac G: Molecular approaches to the study of dermatophytes. Med Mycol 38: 329-336, 2000.

4. Shehata AS, Mukherjee PK, Aboulatta HN, et al: Single-step PCR using (GACA) 4 primer: utility for rapid identification of dermatophyte species and strains. J Clin Microbiol 46: 2641-2645, 2008.

5. Zhu H, Wen H and Liao W: Identification of Trichophyton rubrum by PCR fingerprinting. Chin Med J (Engl) 115: 1218-1220, 2002.

6. Orsini M and Romano-Spica V: A microwave-based method for nucleic acid isolation from environmental samples. Lett Appl Microbiol 33: 17-20, 2001. 
7. Cassago A, Panepucci R, Baião A and Henrique-Silva F: Cellophane based mini-prep method for DNA extraction from the filamentous fungus Trichoderma reesei. BMC Microbiol 2: 14, 2002.

8. Kac G, Bougnoux ME, Feuilhade De Chauvin M, Sene S and Derouin F: Genetic diversity among Trichophyton mentagrophytes isolates using random amplified polymorphic DNA method. Br J Dermatol 140: 839-844, 1999.

9. Summerbell RC, Haugland RA, Li A and Gupta AK: rRNA gene internal transcribed spacer 1 and 2 sequences of asexual, anthropophilic dermatophytes related to Trichophyton rubrum. J Clin Microbiol 37: 4005-4011, 1999.

10. Makimura K, Mochizuki T, Hasegawa A, et al: Phylogenetic classification of Trichophyton mentagrophytes complex strains based on DNA sequences of nuclear ribosomal internal transcribed spacer 1 regions. J Clin Microbiol 36: 2629-2633, 1998.

11. Apodaca GandMcKerrow JH:Regulation of Trichophyton rubrum proteolytic activity. Infect Immun 57: 3081-3090, 1989.

12. Jackson CJ, Barton RC, Kelly SL and Evans EG: Strain identification of Trichophyton rubrum by specific amplification of subrepeat elements in the ribosomal DNA nontranscribed spacer. J Clin Microbiol 38: 4527-4534, 2000.

13. Mochizuki T, Kawasaki M, Ishizaki H, et al: Molecular epidemiology of Arthroderma benhamiae, an emerging pathogen of dermatophytoses in Japan, by polymorphisms of the non-transcribed spacer region of the ribosomal DNA. J Dermatol Sci 27: 14-20, 2001.

14. Zhu HM, Liao WQ and Dai JX: PCR fingerprint analysis of Trichophyton rubrum and Trichophyton mentagrophytes. Chin J Dermatol 33: 349, 2000 (In Chinese).
15. Shin JH, Sung JH, Park SJ, et al: Species identification and strain differentiation of dermatophyte fungi using polymerase chain reaction amplification and restriction enzyme analysis. J Am Acad Dermatol 48: 857-865, 2003

16. Li Q, Liu WD, Yang GL and Li CJ: DNA typing of Trichophyton rubrum. Chin J Dermatol 35: 352-354, 2002 (In Chinese).

17. Jackson CJ, Mochizuki T and Barton RC. PCR fingerprinting of Trichophyton mentagrophytes var. interdigitale using polymorphic subrepeat loci in the rDNA nontranscribed spacer. J Med Microbiol 55: 1349-1355, 2006.

18. Takeda K, Nishibu A, Anzawa K and Mochizuki T: Molecular epidemiology of a major subgroup of Arthroderma benhamiae isolated in Japan by restriction fragment length polymorphism analysis of the non-transcribed spacer region of ribosomal RNA gene. Jpn J Infect Dis 65: 233-239, 2012.

19. Fan JF, Li HJ, Suo JJ, Li RY and Wan Z: Strain typing in Trichophyton rubrum. Academic Journal of Chinese Pla Medical School 25: 225-226, 2004 (In Chinese).

20. Nishio K, Kawasaki M and Ishizaki H: Phylogeny of the genera Trichophyton using mitochondrial DNA analysis. Mycopathologia 117: 127-132, 1992.

21. Jackson CJ, Barton RC and Evans EG: Species identification and strain differentiation of dermatophyte fungi by analysis of ribosomal-DNA intergenic spacer regions. J Clin Microbiol 37: 931-936, 1999. 